UWThPh-2000-10

February 2000

\title{
Gravitational anomalies in a dispersive approach*
}

\author{
R.A. Bertlmann and E. Kohlprath ${ }^{\dagger}$ \\ Institut für Theoretische Physik, Universität Wien \\ Boltzmanngasse 5, A-1090 Vienna, Austria
}

\begin{abstract}
The gravitational anomalies in two dimensions, specifically the Einstein anomaly and the Weyl anomaly, are fully determined by means of dispersion relations. In this approach the anomalies originate from the peculiar infrared feature of the imaginary part of the relevant formfactor which approaches a $\delta$-function singularity at zero momentum squared when $m \rightarrow 0$.
\end{abstract}

\section{INTRODUCTION}

Anomalies are the key to a deeper understanding of quantum field theory. Since their first discovery by Adler [国, Bell and Jackiw [2], and by Bardeen [3] they play a fundamental role in physics (for details see Ref. 四). Also in gravitation, where fermions interact with a gravitational field, anomalies may occur. These are the Einstein anomaly - signaling the breakdown of energy-momentum conservation - the Lorentz anomaly - reflecting an antisymmetric part of the energy-momentum tensor - and the Weyl anomaly - expressing the nonvanishing tensor trace. We mainly refer to the work of the authors [5] - [11 who have calculated (ultraviolet divergent) Feynman diagrams where the external gravitational field couples to a fermion loop via the energy-momentum tensor.

In our paper we want to draw attention to an other approach with interesting features. It is the dispersion relation (DR) approach which is an independent and complementary view of the anomaly phenomenon as compared to the ultraviolet regularization procedures.

${ }^{*}$ This work was partly supported by Austria-Czech Republic Scientific collaboration, project KONTACT 1999-8.

†Supported by a Wissenschaftsstipendium der Magistratsabteilung 18 der Stadt Wien. 
In connection with anomalies DR have been introduced by Dolgov and Zakharov 12 and also by Kummer [13]. In the following several authors [14] - [17] used successfully DR to determine the anomalies in the chiral current. Recently Hořejší and Schnabl [18] have applied the method to the well-known trace anomaly which is related to the broken dilatation (or scale) invariance. We extend in our work the method of DR to the case of pure gravitation and perform the calculations in two dimensions.

\section{STRUCTURE OF THE AMPLITUDE}

The gravitational anomalies are determined by the one-loop diagram of a Weyl fermion in a gravitational background field. Since it is sufficient to work with a linearized gravitational field $g_{\mu \nu}=\eta_{\mu \nu}+\kappa h_{\mu \nu}$ and $e_{\mu}^{a}=\eta_{\mu}^{a}+\frac{1}{2} \kappa h^{a}{ }_{\mu}$, where $e_{\mu}^{a}$ is the zweibein and $E_{a}^{\mu}$ its inverse $E_{a}{ }^{\mu} e_{\nu}^{a}=\delta_{\nu}^{\mu}$, we can start with the following linearized interaction Lagrangian (for convenience $\kappa$ is absorbed into $h^{a \mu}, \partial_{\mu}^{\psi}$ acts only on $\psi$ )

$$
\mathcal{L}_{I}^{l i n}=-\frac{i}{4}\left(h^{a \mu} \bar{\psi} \gamma_{a} \frac{1 \pm \gamma_{5}}{2} \stackrel{\leftrightarrow}{\partial_{\mu}^{\psi}} \psi+h_{\mu}^{\mu}{ }_{\mu} \bar{\psi} \gamma^{a} \frac{1 \pm \gamma_{5}}{2} \stackrel{\leftrightarrow}{\partial_{a}^{\psi}} \psi\right)=-\frac{1}{2} h_{\mu \nu} T^{\mu \nu} .
$$

From this expression follow the Feynman rules for the vertices in the loop and the explicit form of the (symmetric) energy-momentum tensor

$$
\begin{aligned}
T^{\mu \nu} & =\frac{1}{2}\left(T_{a}^{\mu} E^{a \nu}+T_{a}^{\nu} E^{a \mu}\right) \\
& =\frac{i}{4}\left(\bar{\psi} E^{a \nu} \gamma_{a} \frac{1 \pm \gamma_{5}}{2} \stackrel{\leftrightarrow}{\partial^{\mu}} \psi+\bar{\psi} E^{a \mu} \gamma_{a} \frac{1 \pm \gamma_{5}}{2} \stackrel{\leftrightarrow}{\partial^{\nu}} \psi\right)
\end{aligned}
$$

(note that in two dimensions the spin connection $\omega_{\mu}$ does not contribute, see e.g. Ref. 画).

Then the whole amplitude under consideration is given by the two-point function

$$
T_{\mu \nu \rho \sigma}(p)=i \int d^{2} x e^{i p x}\left\langle 0\left|T\left[T_{\mu \nu}(x) T_{\rho \sigma}(0)\right]\right| 0\right\rangle .
$$

Due to Lorentz covariance and symmetry we decompose and separate the amplitude in the following way

$$
\begin{gathered}
T_{\mu \nu \rho \sigma}=T_{\mu \nu \rho \sigma}^{V}+T_{\mu \nu \rho \sigma}^{A} \\
T_{\mu \nu \rho \sigma}^{V}(p)=p_{\mu} p_{\nu} p_{\rho} p_{\sigma} T_{1}\left(p^{2}\right)+\left(p_{\mu} p_{\nu} g_{\rho \sigma}+p_{\rho} p_{\sigma} g_{\mu \nu}\right) T_{2}\left(p^{2}\right) \\
+\left(p_{\mu} p_{\rho} g_{\nu \sigma}+p_{\mu} p_{\sigma} g_{\nu \rho}+p_{\nu} p_{\rho} g_{\mu \sigma}+p_{\nu} p_{\sigma} g_{\mu \rho}\right) T_{3}\left(p^{2}\right) \\
+g_{\mu \nu} g_{\rho \sigma} T_{4}\left(p^{2}\right)+\left(g_{\mu \rho} g_{\nu \sigma}+g_{\mu \sigma} g_{\nu \rho}\right) T_{5}\left(p^{2}\right) \\
T_{\mu \nu \rho \sigma}^{A}(p)=\left(\varepsilon_{\mu \tau} p^{\tau} p_{\nu} p_{\rho} p_{\sigma}+\varepsilon_{\nu \tau} p^{\tau} p_{\mu} p_{\rho} p_{\sigma}+\varepsilon_{\rho \tau} p^{\tau} p_{\mu} p_{\nu} p_{\sigma}+\varepsilon_{\sigma \tau} p^{\tau} p_{\mu} p_{\nu} p_{\rho}\right) T_{6}\left(p^{2}\right) \\
+\left(\varepsilon_{\mu \tau} p^{\tau} p_{\nu} g_{\rho \sigma}+\varepsilon_{\nu \tau} p^{\tau} p_{\mu} g_{\rho \sigma}+\varepsilon_{\rho \tau} p^{\tau} p_{\sigma} g_{\mu \nu}+\varepsilon_{\sigma \tau} p^{\tau} p_{\rho} g_{\mu \nu}\right) T_{7}\left(p^{2}\right) \\
+\left[\varepsilon_{\mu \tau} p^{\tau}\left(p_{\rho} g_{\nu \sigma}+p_{\sigma} g_{\nu \rho}\right)+\varepsilon_{\nu \tau} p^{\tau}\left(p_{\rho} g_{\mu \sigma}+p_{\sigma} g_{\mu \rho}\right)\right. \\
\left.+\varepsilon_{\rho \tau} p^{\tau}\left(p_{\mu} g_{\nu \sigma}+p_{\nu} g_{\mu \sigma}\right)+\varepsilon_{\sigma \tau} p^{\tau}\left(p_{\mu} g_{\nu \rho}+p_{\nu} g_{\mu \rho}\right)\right] T_{8}\left(p^{2}\right) .
\end{gathered}
$$


The functions $T_{1}\left(p^{2}\right), \ldots, T_{8}\left(p^{2}\right)$ are the formfactors that are to be evaluated.

The classical properties of the energy-momentum tensor $\left(T_{\mu \nu}=T_{\nu \mu}\right.$ symmetric, $\nabla^{\mu} T_{\mu \nu}=0$ conserved, $T_{\mu}^{\mu}=0$ traceless) lead to the following canonical (naive) Ward identities:

1. $T_{\mu \nu \rho \sigma}(p)=T_{\nu \mu \rho \sigma}(p)$

2. $p^{\mu} T_{\mu \nu \rho \sigma}(p)=0$

3. $g^{\mu \nu} T_{\mu \nu \rho \sigma}(p)=0$.

We are interested in the pure Einstein anomaly therefore we demand the quantized energy-momentum tensor to be symmetric, which is always possible to achieve. Thus the symmetry property 1.) of the amplitude is fulfilled, however, the Ward identity (WI) 2.) and the trace identity (TI) 3.) need not be satisfied, they can be broken by the Einsteinand the Weyl anomaly respectively.

The canonical Ward identities we re-express by the formfactors. For the pure tensor part of the amplitude the WI may be written as

$$
\begin{aligned}
& p^{2} T_{1}+T_{2}+2 T_{3}=0 \\
& p^{2} T_{2}+T_{4}=0 \\
& p^{2} T_{3}+T_{5}=0
\end{aligned}
$$

and the TI as

$$
p^{2} T_{1}+2 T_{2}+4 T_{3}=0 .
$$

In the following we shall use a renormalization procedure which keeps the WI in the pure tensor part (2.7) - (2.9) so that the anomaly occurs only in the pseudotensor part of the amplitude.

For convenience we split the pure tensor piece of the loop in the following way

$$
T_{\mu \nu \rho \sigma}^{V}(p)=\frac{1}{2} T_{\mu \nu \rho \sigma}^{p v}(p)-T_{\mu \nu \rho \sigma}^{d v}(p),
$$

where $T_{\mu \nu \rho \sigma}^{p v}$ represents the loop with the identity instead of the chirality projectors and $T_{\mu \nu \rho \sigma}^{d v}$ denotes the part proportional to $m^{2}$.

Finally, the axial part of the amplitude is connected to the vector part due to relation

$$
\gamma_{\mu} \gamma_{5}=-\varepsilon_{\mu \nu} \gamma^{\nu}
$$

(valid only in 2 dimensions) where our conventions are $g_{00}=-g_{11}=1, \varepsilon^{01}=1$ and $\gamma^{0}=\sigma^{2}$, $\gamma^{1}=i \sigma^{1}, \gamma^{5}=\gamma^{0} \gamma^{1}=\sigma^{3}$, and $\sigma^{i}$ being the Pauli matrices. 


\section{DISPERSION RELATIONS}

The formfactors of the amplitude $(2.4)$ - (2.6) can be expressed by dispersion relations which relate the real part of the amplitude to its imaginary part. The imaginary parts of the amplitude can be easily calculated via Cutkosky's rule [19. In this way we find the imaginary parts of all formfactors in the total amplitude $T_{\mu \nu \rho \sigma}$ (for details see Ref. [20]):

$$
\begin{aligned}
& \operatorname{Im} T_{1}\left(p^{2}\right)=-\frac{1}{4} J_{0} \frac{m^{2}}{p^{2}}\left(1-4 \frac{m^{2}}{p^{2}}\right) \\
& \operatorname{Im}_{2}\left(p^{2}\right)=-\frac{1}{48} J_{0} p^{2}\left(1-8 \frac{m^{2}}{p^{2}}+16 \frac{m^{4}}{p^{4}}\right) \\
& \operatorname{ImT}_{3}\left(p^{2}\right)=\frac{1}{96} J_{0} p^{2}\left(1+\frac{m^{2}}{p^{2}}-20 \frac{m^{4}}{p^{4}}\right) \\
& \operatorname{ImT}_{4}\left(p^{2}\right)=\frac{1}{48} J_{0} p^{4}\left(1-8 \frac{m^{2}}{p^{2}}+16 \frac{m^{4}}{p^{4}}\right) \\
& \operatorname{Im}_{5}\left(p^{2}\right)=-\frac{1}{96} J_{0} p^{4}\left(1-2 \frac{m^{2}}{p^{2}}-8 \frac{m^{4}}{p^{4}}\right) \\
& \operatorname{Im}_{6}\left(p^{2}\right)= \pm \frac{1}{16} J_{0} \frac{m^{2}}{p^{2}}\left(1-4 \frac{m^{2}}{p^{2}}\right) \\
& \operatorname{ImT}_{7}\left(p^{2}\right)= \pm \frac{1}{192} J_{0} p^{2}\left(1-8 \frac{m^{2}}{p^{2}}+16 \frac{m^{4}}{p^{4}}\right) \\
& \operatorname{Im}_{8}\left(p^{2}\right)=\mp \frac{1}{384} J_{0} p^{2}\left(1+4 \frac{m^{2}}{p^{2}}-32 \frac{m^{4}}{p^{4}}\right)
\end{aligned}
$$

with the threshold function

$$
J_{0}=\frac{1}{p^{2}}\left(1-\frac{4 m^{2}}{p^{2}}\right)^{-1 / 2} \theta\left(p^{2}-4 m^{2}\right) .
$$

Considering on the other hand the amplitude $T_{\mu \nu \rho \sigma}^{p v}$ we get the following imaginary parts:

$$
\begin{aligned}
& \operatorname{Im} A_{1}\left(p^{2}\right)=-\frac{1}{2} J_{0} \frac{m^{2}}{p^{2}}\left(1-4 \frac{m^{2}}{p^{2}}\right) \\
& \operatorname{Im} A_{2}\left(p^{2}\right)=-\frac{1}{24} J_{0} p^{2}\left(1-8 \frac{m^{2}}{p^{2}}+16 \frac{m^{4}}{p^{4}}\right) \\
& I m A_{3}\left(p^{2}\right)=\frac{1}{48} J_{0} p^{2}\left(1+4 \frac{m^{2}}{p^{2}}-32 \frac{m^{4}}{p^{4}}\right) \\
& \operatorname{Im} A_{4}\left(p^{2}\right)=\frac{1}{24} J_{0} p^{4}\left(1-8 \frac{m^{2}}{p^{2}}+16 \frac{m^{4}}{p^{4}}\right) \\
& \operatorname{Im} A_{5}\left(p^{2}\right)=-\frac{1}{48} J_{0} p^{4}\left(1+4 \frac{m^{2}}{p^{2}}-32 \frac{m^{4}}{p^{4}}\right) \text {. }
\end{aligned}
$$

Clearly, the imaginary parts (3.10) - (3.14) of the amplitude $T_{\mu \nu \rho \sigma}^{p v}$ satiesfy the WI (2.7) (2.9) with $T_{i} \rightarrow \operatorname{Im} A_{i}\left(p^{2}\right)$, and the subtraction procedure we choose in the following keeps 
this property for the entire formfactors $A_{i}\left(p^{2}\right)$.

Now we start with an unsubtracted dispersion relation for the formfactors

$$
T\left(p^{2}\right)=\frac{1}{\pi} \int_{4 m^{2}}^{\infty} \frac{d t}{t-p^{2}} \operatorname{Im} T(t)
$$

and we observe that, for instance, the integral for $T_{1}\left(p^{2}\right)$ is convergent whereas for $T_{2}\left(p^{2}\right)$ it is logarithmically divergent and needs to be subtracted once, and for $T_{4}\left(p^{2}\right)$ it is linearly divergent and needs to be subtracted twice. We can infer already from the $p^{2}=t$ behaviour of the imaginary parts which kind of dispersion relation we have to use.

So for the formfactors $T_{1}, T_{6}, A_{1}$ an unsubtracted DR is sufficient and we get

$$
\begin{aligned}
T_{1}\left(p^{2}\right) & =\mp 4 T_{6}\left(p^{2}\right)=\frac{1}{2} A_{1}\left(p^{2}\right) \\
& =-\frac{1}{4 \pi} \int_{4 m^{2}}^{\infty} \frac{d t}{t-p^{2}} \frac{m^{2}}{t^{2}}\left(1-\frac{4 m^{2}}{t}\right)^{\frac{1}{2}} \\
& =\frac{1}{p^{2}}\left[\frac{1}{24 \pi}-\frac{1}{2 \pi} \frac{m^{2}}{p^{2}}+\frac{1}{2 \pi} \frac{m^{2}}{p^{2}} a\left(p^{2}\right)\right]
\end{aligned}
$$

with

$$
a\left(p^{2}\right)=\sqrt{\frac{4 m^{2}-p^{2}}{p^{2}}} \arctan \sqrt{\frac{p^{2}}{4 m^{2}-p^{2}}} .
$$

A once subtracted DR defined by

$$
T^{R}\left(p^{2}\right)=T\left(p^{2}\right)-T(0)=\frac{p^{2}}{\pi} \int_{4 m^{2}}^{\infty} \frac{d t}{t-p^{2}} \frac{1}{t} \operatorname{Im} T(t)
$$

we use for the following formfactors

$$
\begin{aligned}
T_{2}^{R}\left(p^{2}\right) & =\mp 4 T_{7}^{R}\left(p^{2}\right)=\frac{1}{2} A_{2}^{R}\left(p^{2}\right) \\
& =\frac{p^{2}}{\pi} \int_{4 m^{2}}^{\infty} \frac{d t}{t-p^{2}} \frac{1}{t^{2}}\left(1-\frac{4 m^{2}}{t}\right)^{-\frac{1}{2}}\left(-\frac{1}{48} t+\frac{1}{6} m^{2}-\frac{1}{3} \frac{m^{4}}{t}\right) \\
& =-\frac{1}{18 \pi}+\frac{1}{6 \pi} \frac{m^{2}}{p^{2}}+\frac{1}{24 \pi}\left(1-4 \frac{m^{2}}{p^{2}}\right) a\left(p^{2}\right) \\
T_{3}^{R}\left(p^{2}\right) & =\frac{p^{2}}{\pi} \int_{4 m^{2}}^{\infty} \frac{d t}{t-p^{2}} \frac{1}{t^{2}}\left(1-\frac{4 m^{2}}{t}\right)^{-\frac{1}{2}}\left(\frac{1}{96} t+\frac{1}{96} m^{2}-\frac{5}{24} \frac{m^{4}}{t}\right) \\
& =\frac{7}{576 \pi}+\frac{5}{48 \pi} \frac{m^{2}}{p^{2}}-\frac{1}{48 \pi}\left(1+5 \frac{m^{2}}{p^{2}}\right) a\left(p^{2}\right)
\end{aligned}
$$




$$
\begin{aligned}
A_{3}^{R}\left(p^{2}\right) & =\frac{p^{2}}{\pi} \int_{4 m^{2}}^{\infty} \frac{d t}{t-p^{2}} \frac{1}{t^{2}}\left(1-\frac{4 m^{2}}{t}\right)^{-\frac{1}{2}}\left(\frac{1}{48} t+\frac{1}{12} m^{2}-\frac{2}{3} \frac{m^{4}}{t}\right) \\
& =\frac{1}{72 \pi}+\frac{1}{3 \pi} \frac{m^{2}}{p^{2}}-\frac{1}{24 \pi}\left(1+8 \frac{m^{2}}{p^{2}}\right) a\left(p^{2}\right) \\
T_{8}^{R}\left(p^{2}\right) & =\mp \frac{p^{2}}{\pi} \int_{4 m^{2}}^{\infty} \frac{d t}{t-p^{2}} \frac{1}{t^{2}}\left(1-\frac{4 m^{2}}{t}\right)^{-\frac{1}{2}}\left(\frac{1}{384} t+\frac{1}{96} m^{2}-\frac{1}{12} \frac{m^{4}}{t}\right) \\
& =\mp \frac{1}{576 \pi} \mp \frac{1}{24 \pi} \frac{m^{2}}{p^{2}} \pm \frac{1}{192 \pi}\left(1+8 \frac{m^{2}}{p^{2}}\right) a\left(p^{2}\right) .
\end{aligned}
$$

For the remaining formfactors a twice subtracted DR defined by

$$
T^{R}\left(p^{2}\right)=T\left(p^{2}\right)-T(0)-\left.p^{2} \frac{d}{d p^{2}} T\left(p^{2}\right)\right|_{p^{2}=0}=\frac{p^{4}}{\pi} \int_{4 m^{2}}^{\infty} \frac{d t}{t-p^{2}} \frac{1}{t^{2}} \operatorname{Im} T(t)
$$

is necessary and we find

$$
\begin{aligned}
T_{4}^{R}\left(p^{2}\right) & =\frac{1}{2} A_{4}^{R}\left(p^{2}\right)=\frac{p^{4}}{\pi} \int_{4 m^{2}}^{\infty} \frac{d t}{t-p^{2}} \frac{1}{t^{3}}\left(1-\frac{4 m^{2}}{t}\right)^{-\frac{1}{2}}\left(\frac{1}{48} t^{2}-\frac{1}{6} t m^{2}+\frac{1}{3} m^{4}\right) \\
& =p^{2}\left[\frac{1}{18 \pi}-\frac{1}{6 \pi} \frac{m^{2}}{p^{2}}-\frac{1}{24 \pi}\left(1-4 \frac{m^{2}}{p^{2}}\right) a\left(p^{2}\right)\right] \\
T_{5}^{R}\left(p^{2}\right) & =\frac{p^{4}}{\pi} \int_{4 m^{2}}^{\infty} \frac{d t}{t-p^{2}} \frac{1}{t^{3}}\left(1-\frac{4 m^{2}}{t}\right)^{-\frac{1}{2}}\left(-\frac{1}{96} t^{2}+\frac{1}{48} t m^{2}+\frac{1}{12} m^{4}\right) \\
& =p^{2}\left[-\frac{5}{288 \pi}-\frac{1}{24 \pi} \frac{m^{2}}{p^{2}}+\frac{1}{48 \pi}\left(1+2 \frac{m^{2}}{p^{2}}\right) a\left(p^{2}\right)\right] \\
A_{5}^{R}\left(p^{2}\right) & =\frac{p^{4}}{\pi} \int_{4 m^{2}}^{\infty} \frac{d t}{t-p^{2}} \frac{1}{t^{3}}\left(1-\frac{4 m^{2}}{t}\right)^{-\frac{1}{2}}\left(-\frac{1}{48} t^{2}-\frac{1}{12} t m^{2}+\frac{2}{3} m^{4}\right) \\
& =p^{2}\left[-\frac{1}{72 \pi}-\frac{1}{3 \pi} \frac{m^{2}}{p^{2}}+\frac{1}{24 \pi}\left(1+8 \frac{m^{2}}{p^{2}}\right) a\left(p^{2}\right)\right] .
\end{aligned}
$$

With these explicit expressions for the formfactors we have determined the whole amplitude $T_{\mu \nu \rho \sigma}$, Eqs.(2.3)-(2.6), from which the correct Ward identities will follow.

\section{ANOMALOUS WARD IDENTITIES AND GRAVITATIONAL ANOMALIES}

Now we turn to the calculation of the Ward identities and gravitational anomalies. We consider the massless limit, $m \rightarrow 0$, where the formfactors $2 T_{i} \rightarrow A_{i}(\mathrm{i}=1, \ldots, 5)$ fulfill the WI (2.7) - (2.9). This means that the WI for the pure tensor part is satiesfied 


$$
p^{\mu} T_{\mu \nu \rho \sigma}^{V}(p)=0
$$

Next we calculate the WI for the pseudo tensor part. We use the formfactor identities

$$
T_{6}=\mp \frac{1}{4} T_{1}, \quad T_{7}=\mp \frac{1}{4} T_{2}, \quad T_{8}=\mp \frac{1}{4} T_{3},
$$

and we obtain the anomalous result

$$
p^{\mu} T_{\mu \nu \rho \sigma}^{A}(p)=\mp \frac{1}{4} p^{2} T_{1} \varepsilon_{\nu \tau} p^{\tau}\left(p_{\rho} p_{\sigma}-g_{\rho \sigma} p^{2}\right) .
$$

The anomalous WI depends only on the finite formfactor $T_{1}=\mp 4 T_{6}$ with its explicit result

$$
T_{1}\left(p^{2}\right)=\mp 4 T_{6}\left(p^{2}\right)=\frac{1}{24 \pi p^{2}} .
$$

So the anomaly is independent of a specific renormalization procedure (as long as it preserves the WI (2.7)-(2.9) ) and we agree with the anomaly results of Tomiya [21] and Alvarez-Gaumé and Witten [8] who applied quite different (regularization) methods.

We want to emphasize that our subtraction procedure is the 'natural' choice dictated by the $t$-behaviour of the imaginary parts $\operatorname{Im} T_{i}(t)$ of the formfactors, which automatically shifts the total anomaly into the pseudotensor part of the WI (4.3).

What is the origin of the anomaly in this dispersive approach? The source of the anomaly is the existence of a superconvergence sum rule for the imaginary part of the formfactor $T_{1}\left(p^{2}\right)$

$$
\int_{0}^{\infty} d t \operatorname{Im} T_{1}(t)=-\frac{m^{2}}{4} \int_{4 m^{2}}^{\infty} \frac{d t}{t^{2}}\left(1-\frac{4 m^{2}}{t}\right)^{\frac{1}{2}}=-\frac{1}{24}
$$

The anomaly originates from a $\delta$-function singularity of $\operatorname{Im} T_{1}(t)$ when the threshold $t=$ $4 m^{2} \rightarrow 0$ approaches zero (the infrared region)

$$
\lim _{m \rightarrow 0} \operatorname{Im} T_{1}(t)=-\lim _{m \rightarrow 0} \frac{m^{2}}{4 t^{2}}\left(1-\frac{4 m^{2}}{t}\right)^{\frac{1}{2}} \theta\left(t-4 m^{2}\right)=-\frac{1}{24} \delta(t) .
$$

The limit must be performed in a distributional sense.

Then the unsubtracted dispersion relation for $T_{1}\left(p^{2}\right)$, Eq.(3.15), provides the result (4.4). This threshold singularity of the imaginary part of the relevant formfactor is a typical feature of the DR approach for calculating the anomaly (see e.g. Refs. [4], [14] - [18]).

Next we turn to the energy-momentum tensor. From the anomalous WI (4.3) we can deduce the linearized consistent Einstein (or diffeomorphism) anomaly

$$
\partial^{\mu}\left\langle T_{\mu \nu}\right\rangle=\mp \frac{1}{192 \pi} \varepsilon_{\mu \nu} \partial^{\mu}\left(\partial_{\alpha} \partial_{\beta} h^{\alpha \beta}-\partial_{\alpha} \partial^{\alpha} h_{\beta}^{\beta}\right) \text {. }
$$


Result (4.7) is indeed the linearization of the exact result that follows from differential geometry and topology (see for instance Ref. [4]).

Now what about the covariant Einstein anomaly? It arises when considering the covariantly transforming energy-momentum tensor $\tilde{T}_{\mu \nu}$ which is related to our tensor definition (2.2) by the Bardeen-Zumino polynomial $\mathcal{P}_{\mu \nu}[22]$

$$
\left\langle\tilde{T}_{\mu \nu}\right\rangle=\left\langle T_{\mu \nu}\right\rangle+\mathcal{P}_{\mu \nu}
$$

This polynomial is calculable explicitly, for its linearized version we find

$$
\partial^{\mu} \mathcal{P}_{\mu \nu}=\mp \frac{1}{192 \pi} \varepsilon_{\mu \nu} \partial^{\mu}\left(\partial_{\alpha} \partial_{\beta} h^{\alpha \beta}-\partial_{\alpha} \partial^{\alpha} h_{\beta}^{\beta}\right)
$$

leading to the linearized covariant Einstein anomaly

$$
\partial^{\mu}\left\langle\tilde{T}_{\mu \nu}\right\rangle=\mp \frac{1}{96 \pi} \varepsilon_{\mu \nu} \partial^{\mu}\left(\partial_{\alpha} \partial_{\beta} h^{\alpha \beta}-\partial_{\alpha} \partial^{\alpha} h_{\beta}^{\beta}\right) .
$$

It is twice the linearized consistent result (4.7) as it should be.

Finally we also calculate the trace identity. Using again relations (4.2) and taking into account the WI (2.7) provides us the anomalous result

$$
T_{\mu \rho \sigma}^{\mu}=-p^{2} T_{1}\left[\left(p_{\rho} p_{\sigma}-p^{2} g_{\rho \sigma}\right) \mp \frac{1}{4}\left(\varepsilon_{\rho \lambda} p^{\lambda} p_{\sigma}+\varepsilon_{\sigma \lambda} p^{\lambda} p_{\rho}\right)\right] .
$$

Also the anomalous TI depends only on the finite formfactor $T_{1}=\mp 4 T_{6}$ so that it is independent of a specific renormalization procedure (which preserves the WI (2.7)-(2.9)).

Inserting the formfactor, Eq.(4.4), implies the following linearization of the Weyl (or trace) anomaly

$$
\left\langle T_{\mu}^{\mu}\right\rangle=\frac{1}{48 \pi}\left[\left(\partial_{\mu} \partial_{\nu} h^{\mu \nu}-\partial_{\mu} \partial^{\mu} h_{\nu}^{\nu}\right) \mp \frac{1}{2} \varepsilon_{\mu \lambda} \partial^{\lambda} \partial_{\nu} h^{\mu \nu}\right] .
$$

Again, result (4.12) is indeed the linearization of the exact result (see for instance Ref. [4]).

Adding last but not least the Bardeen-Zumino polynomial $\mathcal{P}_{\mu \nu}$ with its linearization

$$
\mathcal{P}_{\mu}^{\mu}= \pm \frac{1}{96 \pi} \varepsilon^{a b} \partial^{\mu} \partial_{b} h_{\mu a}
$$

we find for the linearized covariant trace anomaly

$$
\left\langle T_{\mu}^{\mu}\right\rangle=\frac{1}{48 \pi}\left(\partial_{\mu} \partial_{\nu} h^{\mu \nu}-\partial_{\mu} \partial^{\mu} h_{\nu}^{\nu}\right)
$$

Clearly this result is in agreement with Ref. [11]. 


\section{CONCLUSIONS}

We have investigated an alternative method, the DR approach, to calculate the gravitational anomalies. The method appears quite appealing, all one has to calculate is the imaginary part of just one formfactor of the amplitude $T_{\mu \nu \rho \sigma}(p)$, namely $\operatorname{Im} T_{1}\left(p^{2}\right)$, which is an easy task.

Our 'natural' subtraction procedure for the formfactors implies that the pure tensor WI (2.7) $-(2.9)$ for the renormalized formfactors is satisfied (in the limit $m \rightarrow 0$ ), so that the total anomaly is automatically shifted into the pseudotensor part of the WI (4.3). From the anomalous WI and the anomalous TI follow the linearized Einstein- and Weyl anomaly, and we have also determined their covariant versions. An analogous dispersive calculation of the anomalous commutators of the energy-momentum tensors - the gravitational Schwinger terms - is given elsewhere [23].

The anomalies originate from the peculiar infrared feature of the imaginary part of the relevant formfactor $T_{1}\left(p^{2}\right)$ which approaches a $\delta$-function singularity at zero momentum squared when $m \rightarrow 0$.

We have considered the anomalies in two dimensions, where the essential features of the DR approach already show up and all calculations come out very simple. However, this convenient computational simplicity is a very special feature of the two space-time dimensions, in higher dimensions the calculations will turn out much more complicated. 


\section{REFERENCES}

[1] S. Adler, Phys. Rev. 177 (1969) 2426.

[2] J.S. Bell and R. Jackiw, Nuovo Cimento 60A (1969) 47.

[3] W.A. Bardeen, Phys. Rev. 184 (1969) 1848.

[4] R.A. Bertlmann, Anomalies in Quantum Field Theory. International series of monographs on physics 91, Clarendon Press, Oxford (1996).

[5] D.M. Capper and M.J. Duff, Nuovo Cimento A 23 (1974) 173.

[6] S. Deser, M.J. Duff and C.J. Isham, Nucl. Phys. B 111 (1976) 45.

[7] S. Coleman and R. Jackiw, Ann. Phys. (NY) 67 (1971) 552.

[8] L. Alvarez-Gaumé and E. Witten, Nucl. Phys. B 234 (1983) 269.

[9] P. van Nieuwenhuizen, Anomalies in quantum field theory: cancellation of anomalies in $d=10$ supergravity. Leuven notes in mathematical and theoretical physics, Vol. 3, Leuven University Press (1988).

[10] F. Langouche, Phys. Lett. 148B (1984) 93.

[11] H. Leutwyler, Phys. Lett. 153B (1985) 65.

[12] A.D. Dolgov and V.I. Zakharov, Nucl. Phys. B 27 (1971) 525.

[13] W. Kummer, Acta Phys. Austr. Suppl. VII (1970) 567.

[14] J. Hořejší, Phys. Rev. D 32 (1985) 1029.

[15] J. Hořejší, Czech. J. Phys. 42 (1992) 241, 42 (1992) 345.

[16] C. Adam, R.A. Bertlmann and P. Hofer, Z. Phys. C 56 (1992) 123.

[17] C. Adam, R.A. Bertlmann and P. Hofer, La Rivista del Nuovo Cimento Vol. 16, ser. 3, N. 8 (1993) 1.

[18] J. Hořejší, M. Schnabl, Z. Phys. C 76 (1997) 561.

[19] R.E. Cutkosky, J. Math. Phys. 1 (1960) 429.

[20] E. Kohlprath, Diffeomorphism anomaly and Schwinger terms in two dimensions, Diploma thesis at the University of Vienna (June 1999).

[21] M. Tomiya, Phys. Lett. 167B (1986) 411.

[22] W.A. Bardeen and B. Zumino, Nucl. Phys. B 244 (1984) 421.

[23] R.A. Bertlmann and E. Kohlprath, Schwinger terms in gravitation and Källen's method, preprint University of Vienna UWThPh-2000-11. 